\title{
A note on the NP-hardness of two matching problems in induced subgrids $\|^{\dagger}$
}

\author{
Marc Demange ${ }^{1,2}$ | and Tinaz Ekim非 \\ ${ }^{1}$ ESSEC Business School, Paris, France. \\ ${ }^{2}$ LAMSADE, UMR CNRS 7243, Paris, France. \\ ${ }^{3}$ Boğaziçi University, Industrial Engineering Department, Turkey.
}

received $11^{\text {th }}$ Apr. 2012, revised $6^{\text {th }}$ Aug. 2013, accepted $9^{\text {th }}$ Aug. 2013.

Given a graph, finding the maximal matching of minimum size (MMM) and the induced matching of maximum size (MIM) have been very popular research topics during the last decades. In this paper, we give new complexity results, namely the NP-hardness of MMM and MIM in induced subgrids and we point out some promising research directions. We also sketch the general framework of a unified approach to show the NP-hardness of some problems in subgrids.

Keywords: Minimum maximal matching, edge dominating set, maximum induced matching, grid graphs

\section{Introduction}

Given a graph $G$, a matching $M$ is a set of edges which are pairwise non-adjacent. A matching is said to be maximal if no other edge can be added to it while keeping the property of it being a matching. Vertices contained in edges of a matching are said to be saturated by this matching. A vertex which is not saturated by a matching is called exposed. The problem of finding a maximal matching of minimum size is called Minimum Maximal Matching (MMM) or Minimum (Independent) Edge Dominating Set (see [26] for the equivalence of these problems). Given a graph $G$, the size of a minimum maximal matching is denoted by $\beta(G)$.

MMM, NP-hard in general, is extensively studied since early 80 s due to its theoretical and practical interest. In [26], Yannakakis and Gavril show that MMM is NP-hard in several classes of graphs including bipartite (or planar) graphs with maximum degree 3. In [14], Horton and Kilakos extended these results by showing the NP-hardness of MMM in planar bipartite graphs and planar cubic graphs. In [28], Zito shows that the problem remains NP-hard in the so-called almost regular bipartite graphs, that are bipartite graphs

\footnotetext{
${ }^{\dagger}$ This research was supported by Grant 108M616 / 22442 of CNRS-TÜBITAK joint research project whose support is greatly acknowledged. M. Demange also acknowledges support by the French Agency for Research under the DEFI program TODO, ANR-09-EMER-010

¥Email: demangedessec.edu

$\S$ Email: tinaz.ekim@boun.edu.tr

1365-8050 @ 2013 Discrete Mathematics and Theoretical Computer Science (DMTCS), Nancy, France
} 
for which the ratio between the maximum degree $\Delta$ and the minimum degree $\delta$ is bounded. Another strengthening of the result of Yannakakis and Gavril in [26] is given in [9] by showing that MMM is NP-hard in $k$-regular bipartite graphs for any fixed $k \geq 3$. On the other hand, polynomial time algorithms for MMM are designed for trees [20], for block graphs [15], for series-parallel graphs [21], for bipartite permutation graphs and cotrianglated graphs [22], and for clique-width bounded graphs [10].

Due to the hardness of solving MMM even in very restricted classes of graphs, many recent works on MMM concentrate on two aspects: the approximation point of view (see e.g. [28]) and the exact resolution of MMM in general graphs via Integer Programming techniques [24, 1].

Another extensively studied problem related to matchings is the problem of finding an induced matching of maximum size, the so-called Maximum Induced Matching (MIM). A matching is induced if no two vertices belonging to different edges of the matching are adjacent. Given a graph $G$, the cardinality of a maximum induced matching is denoted by $i \mu(G)$. MIM, also NP-hard in general, remains NP-hard in several graph classes such as planar graphs of degree at most 4 [16], line graphs [17], bipartite graphs [23], bipartite graphs of girth at least 14 [2], or of girth at least 6 [28] or of degree at most 3 [18]. This last result has been recently improved in [7] by proving that MIM is NP-hard in $k$-regular bipartite graphs for any $k \geq 3$. On the other hand, MIM can be polynomially solved in weakly chordal graphs [4], AT-free graphs [5, 3], circular arc graphs [11], cocomparability graphs [12], graphs of bounded clique-width [17], chordal graphs [2] and interval filament graphs [3], which include cocomparability graphs and polygoncircle graphs, where the latter include circle graphs, circular-arc graphs, chordal graphs, and outerplanar graphs.

In this paper, we contribute to narrowing down the gap between P and NP-complete with respect to MMM by showing that MMM is NP-hard in induced subgrids of degree 2 and 3 with arbitrarily large girth. Starting from the NP-hardness of MMM in cubic planar graphs, we reduce it to the case of subgrids by embedding a planar graph into a grid and studying how a solution is affected by edge subdivisions. Similarly, we show that MIM is NP-hard in induced subgrids of degree 2 and 4 with arbitrarily large girth. This strengthens the result in [28] on the NP-hardness of MIM in bipartite graphs of girth at least 6. A similar thought-process has been used for other problems known to be NP-hard in planar graphs of degree 4; e.g. some list-coloring problems in [8]. In the conclusion, we give a general framework to apply this approach to other problems. We also discuss the tractability of MMM and MIM in grids and point out some open research directions on the topic.

$P_{n}$ denotes a chordless path on $n$ vertices. The length of a path is the number of edges in it. A twodimensional $n \times m$ grid graph (or grid for short) $G_{n, m}=(V, E)$ will have vertex set $V=\left(\left(x_{i}, y_{j}\right), i=\right.$ $1, \ldots, n ; j=1, \ldots, m)$ where $x_{i}=i, y_{j}=j$ for all $i, j$ and edge set $E$ such that $\left(x_{i}, y_{i}\right)\left(x_{i}^{\prime}, y_{i}^{\prime}\right) \in E \Leftrightarrow$ $\left|x_{i}-x_{i}^{\prime}\right|+\left|y_{i}-y_{i}^{\prime}\right|=1$.

An induced subgrid of a grid $G$ is obtained by removing some of its vertices (and hence the edges incident to them) whereas a partial subgrid of $G$ is obtained from an induced subgrid by removing some edges. In this paper, whenever we say "subgrid" without specifying whether it is partial or induced, we mean an induced subgrid.

Given a graph $G$ and weights $W=(w(e), e \in E)$ where $w(e)$ denotes the weight of an edge $e$, let us define $\widetilde{G}_{W}$ as the graph obtained from $G$ by subdividing each of its edges $e$ by $3 w(e)$ vertices; in other words, each edge $e$ of $G$ becomes in $\widetilde{G}_{W}$ a chordless path of length $3 w(e)+1$. In what follows, $M$ and $M^{\prime}$ denote matchings in $G$ whereas $\widetilde{M}$ and $\widetilde{M}^{\prime}$ refer to matchings in $\widetilde{G}_{W}$. When no ambiguity occurs, for any vertex $u$ of $G$, the corresponding vertex in $\widetilde{G}_{W}$ is also denoted by $u$. In related figures, these vertices 
are black whereas additional vertices of $\widetilde{G}_{W}$ are white.

\section{MMM in induced subgrids}

The following lemma states that given a graph $G$ and weights $W$ on its edges, there is a minimum maximal matching of $\widetilde{G}_{W}$ whose edges have only a specific form with respect to the chordless paths of $\widetilde{G}_{W}$ obtained by subdividing the edges of $G$.

Lemma 2.1 Given a graph $G$ and weights $W$ on its edges, there exists a minimum maximal matching $\widetilde{M}$ of $\widetilde{G}_{W}$ such that for each edge $u v \in E(G)$, the corresponding subdivided path $P_{u v}=u, x_{1}, x_{2}, \ldots, x_{3 w(u v)}, v$ in $\widetilde{G}_{W}$ is of one of the following types:

Type 1: $u x_{1}, x_{3 i} x_{3 i+1}(i \in\{1, \ldots, w(u v)-1\}), x_{3 w(u v)} v \in \widetilde{M}$; hence $\widetilde{M}$ has exactly $w(u v)+1$ edges in $P_{u v}$,

Type 2: $u x_{1}, x_{3 w(u v)} v \notin \widetilde{M}, x_{3 i-1} x_{3 i}(i \in\{1, \ldots, w(u v)\}) \in \widetilde{M}$, u and $v$ are saturated by $\widetilde{M} ;$ hence $\widetilde{M}$ has exactly $w(u v)$ edges in $P_{u v}$,

Type 3: $u x_{1} \notin \widetilde{M}, x_{3 i-1} x_{3 i}(i \in\{1, \ldots, w(u v)\}) \in \widetilde{M}$, u is saturated and $v$ is exposed with respect to $\widetilde{M}$; hence $\widetilde{M}$ has exactly $w(u v)$ edges in $P_{u v}$.

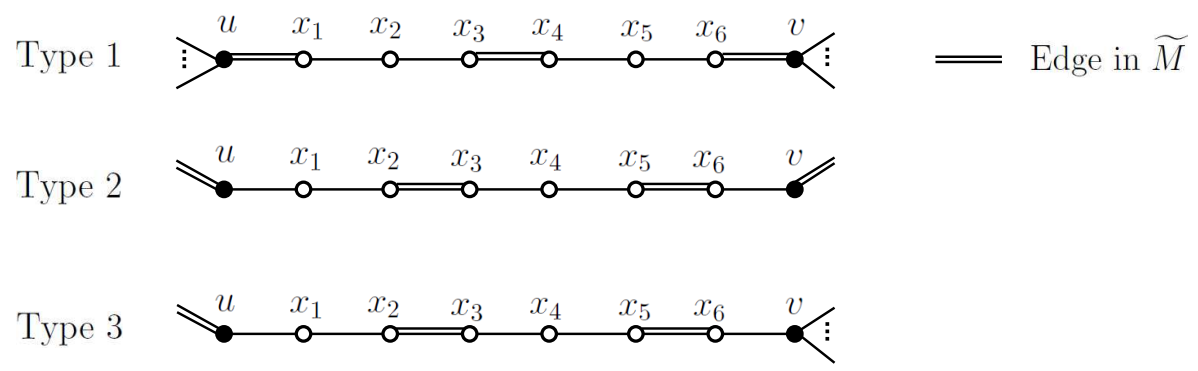

Fig. 1: Possible types for a path $P_{u v}$ corresponding to an edge $u v \in E(G)$ (with $w(u v)=2$ ) in a minimum maximal matching $\widetilde{M}$ of $\widetilde{G}_{W}$.

Proof: For any minimum maximal matching $\widetilde{M}^{\prime}$ in $\widetilde{G}_{W}$, the end vertices of a path $P_{u v}$ can be either both saturated, or both exposed, or one saturated and the other one exposed. Each time $u$ or $v$ is saturated there are still two possibilities: it is saturated either by an edge in $P_{u v}$ or by an edge not in $P_{u v}$. Having Types 1, 2 and 3 as illustrated in Figure 1, there are three remaining cases with respect to the saturation of vertices $u$ and $v$ by $\widetilde{M^{\prime}}$ (illustrated in Figure 2); if there is a maximal matching containing one of these remaining cases for a path $P_{u v}$, then we show in the sequel that this maximal matching can be locally modified without changing its size and without harming its maximality such that $P_{u v}$ becomes of Type 1 , 2 or 3 and no new remaining case is created by the modification. 


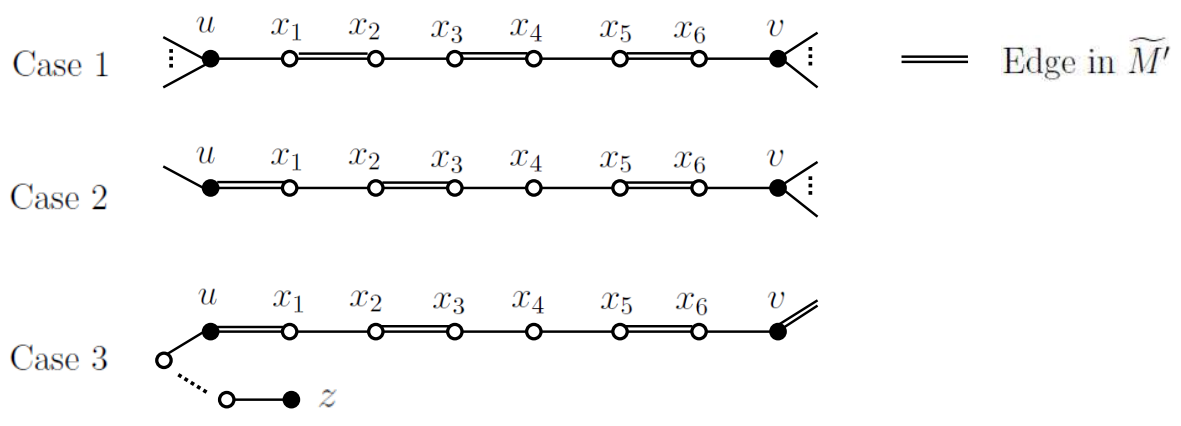

Fig. 2: Remaining cases for a path $P_{u v}$ corresponding to an edge $u v \in E(G)$ (with $w(u v)=2$ ) in a minimum maximal matching $\widetilde{M}^{\prime}$ of $\widetilde{G}_{W}$.

Case 1: Both $u$ and $v$ are exposed.

Then by maximality of $\widetilde{M^{\prime}}$, we have necessarily $x_{1} x_{2} \in \widetilde{M^{\prime}}$ and similarly $x_{3 w(u v)-1} x_{3 w(u v)} \in \widetilde{M}^{\prime}$. The maximality of $\widetilde{M^{\prime}}$ also forces it to contain $w(u v)-1$ additional edges in $P_{u v}$; which sums up to $w(u v)+1$ edges in $P_{u v}$. It can be easily noticed that such a path can be replaced by a path of Type 1 (of the same length); the new matching is still maximal since a path of Type 1 has a maximal matching in $P_{u v}$ and no change is carried out by this replacement in $\widetilde{M^{\prime}}$ in edges not contained in $P_{u v}$. Moreover, the size of the new matching $\widetilde{M}$ is the same as the size of $\widetilde{M^{\prime}}$.

Case 2: $u x_{1} \in \widetilde{M}^{\prime}$ and $v$ is exposed.

Similarly to Case 1 , such a $P_{u v}$ can be replaced by a path of Type 1 , defining a new matching $\widetilde{M}$.

Case 3: $u x_{1} \in \widetilde{M}^{\prime}, x_{3 w(u v)} v \notin \widetilde{M^{\prime}}$ and $v$ is saturated.

The maximality of $\widetilde{M}^{\prime}$ forces it to contain at least $w(u v)+1$ edges in $P_{u v}$. Let us consider $N_{G}(u) \backslash$ $\{v\}$, the neighborhood of $u$ in $G$ and different from $v$. Let us suppose that all vertices $z \in N_{G}(u) \backslash$ $\{v\}$ are saturated in $\widetilde{G}_{W}$ with respect to $\widetilde{M}^{\prime}$ and show that in this case $\widetilde{M}^{\prime}$ would not be of minimum size. In fact, in such a case, if there is $z \in N_{G}(u) \backslash\{v\}$ such that $z$ is saturated by an edge in $P_{u z}$, then $P_{u v}$ can be modified into Type 2 and $P_{u z}$ (from Case 3) into Type 1 (by adding in $\widetilde{M}^{\prime}$ the edge incident to $u$ in $P_{u z}$ and removing from $\widetilde{M^{\prime}}$ one edge in $P_{u z}$ ), the other paths $P_{u z^{\prime}}$ for other neighbors $z^{\prime}$ of $u$ remaining unchanged. Note that the size of the so-obtained maximal matching is one less than the size of $\widetilde{M^{\prime}}$. On the other hand, if for all $z \in N_{G}(u) \backslash\{v\}, z$ is saturated by an edge not in $P_{u z}$, then $P_{u v}$ can be modified into a path of Type 3 (where $u$ becomes exposed) and all the paths $P_{u z}$ are modified (from Type 2) into Type 3. Again, this results in a maximal matching of lower size.

So, there is at least one vertex $z \in N_{G}(u) \backslash\{v\}$ such that $z$ is exposed in $\widetilde{G}_{W}$. But then $P_{u z}$ can be transformed (from Type 3) into Type 1 (hence augmenting the size of $\widetilde{M^{\prime}}$ by one on $P_{u z}$ ) and $P_{u v}$ into Type 2 (hence reducing the size of $\widetilde{M}^{\prime}$ by one on $P_{u v}$ ). As a result, we obtain a new maximal matching $\widetilde{M}$ of the same size. 
In all cases, the new matching $\widetilde{M}$ is a minimum maximal matching satisfying the required conditions.

Proposition 2.2 Given a graph $G$ and weights $W$ on its edges, we have $\beta\left(\widetilde{G}_{W}\right)=\beta(G)+\sum_{e \in E(G)} w(e)$.

Proof: Without loss of generality, let $\widetilde{M}$ be a minimum maximal matching of $\widetilde{G}_{W}$ as described in Lemma 2.1. that is such that for all $u v \in E(G), P_{u v}$ is of Type 1,2 or 3 . Now, we define a matching $M^{\prime}$ of $G$ as follows: $M^{\prime}=\left\{u v \mid P_{u v}\right.$ is of Type 1\}. Let us show that $M^{\prime}$ is maximal; note first that all vertices of $G$ saturated in $\widetilde{G}_{W}$ with respect to $\widetilde{M}$ are also saturated with respect to $M^{\prime}$ since they necessarily belong to some path of Type 1. Moreover, consider a vertex $u$ in $G$ which is exposed with respect to $M^{\prime}$ (and hence with respect to $\widetilde{M}$ as well); all of its neighbors in $G$ are saturated by $\widetilde{M}$ in $\widetilde{G}_{W}$ (since they all necessarily correspond to paths of Type 3) and hence they are also saturated by $M^{\prime}$ in $G$. Consequently, $M^{\prime}$ is maximal. Now, we have $\left|M^{\prime}\right|=\beta\left(\widetilde{G}_{W}\right)-\sum_{e \in E(G)} w(e) \geq \beta(G)$.

Conversely, having a minimum maximal matching $M$ of $G$, we define a matching $\widetilde{M^{\prime}}$ as follows: if $u v \in M$ then $P_{u v}$ is of Type 1 ; if $u v \notin M$ and both $u$ and $v$ are saturated (with respect to $M$ ) then $P_{u v}$ is of Type 2; and finally if $u v \notin M, u$ is saturated and $v$ is exposed with respect to $M$, then $P_{u v}$ is of Type 3. As previously, the maximality of $\widetilde{M}^{\prime}$ follows from its definition and the maximality of $M$. Now, it is enough to observe that $\left|\widetilde{M}^{\prime}\right|=\beta(G)+\sum_{e \in E(G)} w(e) \geq \beta\left(\widetilde{G}_{W}\right)$ to conclude the proof.

Let us recall the following result about the embedding of a given planar graph into a grid:

Theorem 2.3 [27] A planar graph $G$ with maximum degree at most 4 can be embedded in a grid graph $G_{0}$ of polynomial size: the vertices $u$ of $G$ are mapped to vertices $u_{0}$ of $G_{0}$; each edge e $=u v$ of $G$ is mapped to a path $e_{0}$ between $u_{0}$ and $v_{0}$ in $G_{0}$; the intermediate vertices of $e_{0}$ are called internal vertices, they belong to exactly one such path.

Note that by subdividing the edges $e=u v$ of $G$ by the internal vertices in its embedding, we obtain a partial subgrid $G^{\prime}$ of $G_{0}$. The edge $u v \in E(G)$ corresponds to a path of length $\ell(u v)$ in $G^{\prime}$. Having a grid (or induced subgrid, or partial subgrid) $G$, we will also use the notion of a $d$-expansion of $G, d \geq 2$, defined in [8] as the subgrid obtained from $G$ by subdividing each edge by $d-1$ vertices; hence a path of length $\ell$ in $G$ becomes a (chordless) path of length $\ell d$ in its $d$-expansion.

Theorem 2.4 MMM is NP-hard in subgrids of degree 2 and 3 and of arbitrarily large girth.

Proof: Given a cubic planar graph $G$, we will show that, for all $g \geq 3$, there are weights $W$ on the edges of $G$ such that $\widetilde{G}_{W}$ is a subgrid of degree 2 and 3 and of girth at least $g$. Let us first consider an embedding of $G$ into a $p \times q$ grid $G_{0}$ of polynomial size. Consider the partial subgrid $G^{\prime}$ obtained by subdividing the edges of this embedding by the internal vertices of $G_{0}$. Let $k=\left\lceil\frac{g}{48}\right\rceil$; take a $9 k$-expansion of $G^{\prime}$, called $G^{\prime \prime}$. Note that $G^{\prime \prime}$ is a subgrid of a $(9 k(p-1)+1) \times(9 k(q-1)+1)$ grid $G_{1}$. Each edge $u v \in E(G)$, corresponding to a path of length $\ell(u v)$ in $G^{\prime}$, is associated to a path of length $9 k \ell(u v)$ between $u_{0}$ and $v_{0}$ in $G^{\prime \prime}$. Clearly, at least the first nine edges of such a path (starting from $u_{0}$ for instance) are either all horizontal or all vertical. Let us call $u_{0}, x_{1}, x_{2}, x_{3}, x_{4}, x_{5}$ the first vertices of this path; we remove edges $x_{3} x_{4}$ and $x_{4} x_{5}$ from $G^{\prime \prime}$ and replace them by a path of $G_{1}$ of length 6 having two corners as in Figure 3. The graph obtained $\widetilde{G}$ is a subgrid of girth at least $g$ and has vertices of degree 2 and 3 . Now, it is easy to note that the edge $u v$ of $G$ corresponds to a path of length $9 k \ell(u v)+4$ in $\widetilde{G}$. So, by letting $w(u v)=3 k \ell(u v)+1$, we have that $\widetilde{G}$ is isomorphic to $\widetilde{G}_{W}$. 


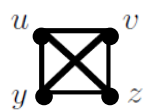

Planar graph $G$ of degree 3

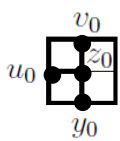

An embedding of $G$ in $3 \times 3$ grid $G_{0}$

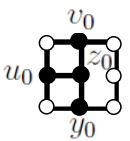

Partial subgrid $G^{\prime}$

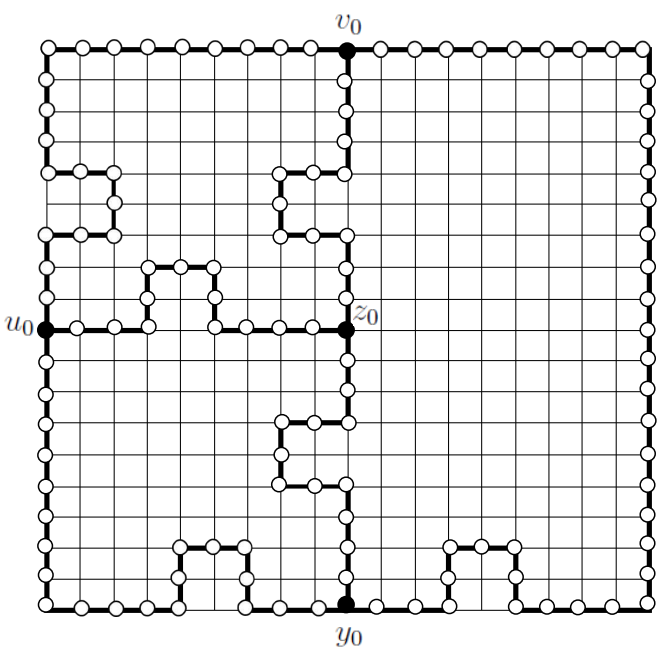

Induced subgrid $\widetilde{G}_{W}$ embedded in $19 \times 19 \operatorname{grid} G_{1}$

Fig. 3: Transformation of a planar graph $G$ into a subgrid $\widetilde{G}_{W}$ of degree 2 and 3 with girth 48 .

Obviously, the above transformation from $G$ to $\widetilde{G}_{W}$ can be made in polynomial time. Moreover, Proposition 2.2 implies that $G$ has a minimum maximal matching of size $\beta(G)$ if and only if there is a minimum maximal matching of size $\beta(G)+\sum_{e \in E(G)} w(e)$ in $\widetilde{G}_{W}$. The proof is concluded by using the NPhardness of MMM in cubic planar graphs [14].

\section{MIM in induced subgrids}

We apply the same methodology to show that MIM is NP-hard in subgrids of degree 2 or 4 and of arbitrary large girth. To this aim, we need the following proposition that is the MIM version of Proposition 2.2

Proposition 3.1 Given a graph $G$ and weights $W$ on its edges, we have $i \mu\left(\widetilde{G}_{W}\right)=i \mu(G)+\sum_{e \in E(G)} w(e)$.

Proof: Let us consider a graph $G$ and weights $W$ on its edges, we first claim that there is a maximum induced matching $\widetilde{M}_{i}$ of $\widetilde{G}_{W}$ such that:

(a) $\forall u v \in E(G),\left|P_{u v} \cap \widetilde{M}_{i}\right| \in\{w(u v), w(u v)+1\}$

(b) If $\left|P_{u v} \cap \widetilde{M}_{i}\right|=w(u v)$ then we have $\widetilde{M}_{i} \cap\left\{u x_{1}, x_{3 w(u v)} v\right\}=\emptyset$ for $P_{u v}=u, x_{1}, x_{2}, \ldots, x_{3 w(u v)}, v$

Let us consider a maximum induced matching $\widetilde{M}_{i}^{\prime}$ of $\widetilde{G}_{W}$. For every $u v \in E(G), P_{u v}$ being of length $3 w(u v)+1$, we have $\left|P_{u v} \cap \widetilde{M}_{i}^{\prime}\right| \leq w(u v)+1$ and moreover if it is equal to $w(u v)+1$ then the matching on this path is of Type 1 as described in Lemma 2.1 (see Figure 1, with $u$ and $v$ saturated by edges of $P_{u v} \cap \widetilde{M}_{i}^{\prime}$. On the other hand, $\widetilde{M}_{i}^{\prime}$ being of maximum size, $\left|P_{u v} \cap M_{i}^{\prime}\right| \geq w(u v)-1$. 
Starting from $\widetilde{M_{i}^{\prime}}$ we will apply successively the two following rules for computing another maximum induced matching $\widetilde{M}_{i}$ satisfying conditions (a) and (b).

We first apply Rule 1 for every edge $u v \in E(G)$ :

Rule 1: If $\left|P_{u v} \cap \widetilde{M}_{i}^{\prime}\right|=w(u v)$ and $\widetilde{M}_{i}^{\prime} \cap\left\{u x_{1}, x_{3 w(u v)} v\right\} \neq \emptyset$, then supposing for instance that $u x_{1} \in \widetilde{M_{i}^{\prime}}$, we replace $\widetilde{M_{i}^{\prime}}$ by $\widetilde{M_{i}^{\prime} \backslash} \backslash\left(P_{u v} \cap \widetilde{M}_{i}^{\prime}\right) \cup\left\{x_{3 i+1} x_{3 i+2}, i=0, \ldots w(u v)-1\right\}$, another maximum induced matching.

Then, for every edge $u v \in E(G)$ such that $\left|P_{u v} \cap \widetilde{M}_{i}^{\prime}\right|=w(u v)-1$, since $\widetilde{M}_{i}^{\prime}$ is maximum, we necessarily have two vertices $z_{1}, z_{2}$ outside $P_{u v}$ such that $z_{1} u \in \widetilde{M_{i}^{\prime}}$ and $v z_{2} \in \widetilde{M}_{i}^{\prime}$. $z_{1}$ belongs to a path $P_{u_{1} u}$ and $z_{2}$ belongs to a path $P_{v u_{2}}$, where $u_{1} u \in E(G), v u_{2} \in E(G)$. Moreover, since Rule 1 has been applied to all edges in $E(G)$ and in particular to $u_{1} u$ and $v u_{2}$, the fact that $z_{1} u \in \widetilde{M_{i}^{\prime}}$ and $v z_{2} \in \widetilde{M_{i}^{\prime}}$ means that $P_{u_{1} u}$ and $P_{v u_{2}}$ have respectively $w\left(u_{1} u\right)+1$ and $w\left(v u_{2}\right)+1$ edges in $\widetilde{M}_{i}^{\prime}$ and therefore they are both of Type 1 .

Rule 2: Replace $\widetilde{M}_{i}^{\prime}$ by $\widetilde{M_{i}^{\prime}} \backslash\left[\left(P_{u v} \cap \widetilde{M}_{i}^{\prime}\right) \cup\left\{v z_{2}\right\}\right] \cup\left\{x_{3 i+2} x_{3 i+3}, i=0, \ldots, w(u v)-1\right\}$ and apply Rule 1 on the path $P_{v u_{2}}$.

Note that when we apply Rule 1 to $P_{v u_{2}}$, it is no more of Type 1 since we first remove $v z_{2}$ from $\widetilde{M}_{i}^{\prime}$. In addition, the other end-edge of $P_{v u_{2}}$ belongs to $\widetilde{M}_{i}^{\prime}$. Thus, Rule 1 can be applied.

Denoting by $\widetilde{M}_{i}$ the transformed induced matching, it is a maximum induced matching satisfying conditions (a) and (b). We then consider the set $M_{i}^{\prime}=\left\{u v \mid P_{u v}\right.$ is of Type 1\}. By condition (a), none of the paths $P_{u v}$ has $\left|P_{u v} \cap \widetilde{M}_{i}\right|=w(u v)-1$ and therefore both $u$ and $v$ cannot simultaneously belong to two different paths of Type 1 . Consequently, $M_{i}^{\prime}$ is an induced matching and moreover $\left|M_{i}^{\prime}\right|=$ $i \mu\left(\widetilde{G}_{W}\right)-\sum_{e \in E(G)} w(e) \leq i \mu(G)$.

Conversely, having a maximum induced matching $M_{i}$ of $G$, we define a matching $\widetilde{M_{i}^{\prime}}$ as follows: if $u v \in M_{i}$ then $P_{u v}$ is of Type 1 ; if $u v \notin M_{i}$, at least one among $u$ and $v$, say $u$ is exposed and then edges $x_{3 i+1} x_{3 i+2}, i=0, \ldots w(u v)-1$ are put in $\widetilde{M}_{i}^{\prime} . \widetilde{M}^{\prime}$ is an induced matching satisfying $\left|\widetilde{M}^{\prime}\right|=$ $i \mu(G)+\sum_{e \in E(G)} w(e) \leq i \mu\left(\widetilde{G}_{W}\right)$, which concludes the proof.

MIM is known to be NP-hard in planar graphs of maximum degree 4 [16]. We can even adapt the proof to show a slightly more restrictive result:

Proposition 3.2 MIM is NP-hard in 4-regular planar graphs.

Proof: The proof of [16] is based on the remark that, given a graph $G$ and adding for each vertex $v$ a vertex $v^{\prime}$ and a pendant edge $v v^{\prime}$, the resulting graph $G^{\prime}$ satisfies $i \mu\left(G^{\prime}\right)=\alpha(G)$, where $\alpha$ denotes the stability number. Moreover if $G$ is planar then $G^{\prime}$ is also planar. NP-hardness in planar graphs of maximum degree 4 ([16]) follows from the fact that Maximum Stable Set problem is NP-hard in cubic planar graphs.

It is shown in [25] that Maximum Stable Set problem remains NP-hard in cubic planar graphs without bridge. Using Petersen's Theorem [13] such graphs admit a perfect matching and consequently:

Lemma 3.3 Max Stable Set problem is NP-hard in cubic planar graphs admitting a perfect matching.

We are now ready to describe the reduction: starting from a planar cubic graph with a perfect matching $M$ we add to each edge $u v \in M$ the gadget $H$ as depicted on Figure 4 


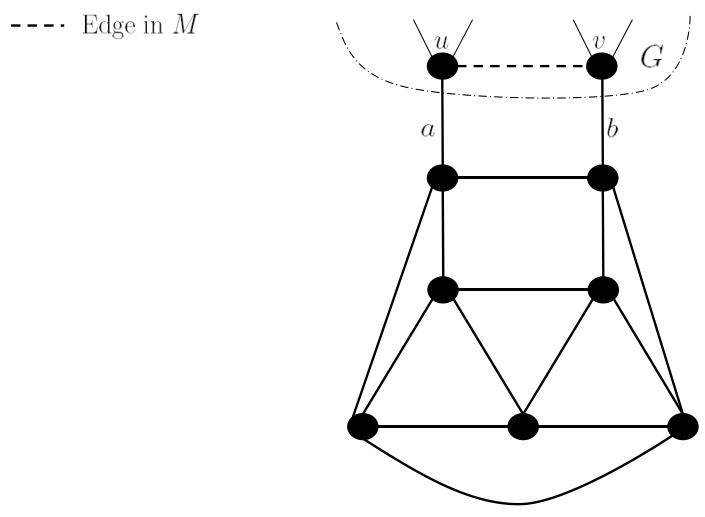

Fig. 4: The gadget $H$.

Denoting by $G^{\prime}$ the resulting graph, it is straightforward to verify that $G^{\prime}$ is planar and 4-regular. Moreover, for any edge $u v \in M$, denote by $E_{H, u, v}$ the set of edges of the copy of the gadget $H$ attached to $u$ and $v$ and denote by $a$ and $b$ the two edges of $E_{H, u, v}$ incident, in $G^{\prime}$, to $u$ and $v$ respectively. We have, for any maximum induced matching $M_{i}^{\prime}$ of $G^{\prime}:\left|M_{i}^{\prime} \cap\{a, b\}\right| \leq 1$ and $\left|M_{i}^{\prime} \cap\left(E_{H, u, v} \backslash\{a, b\}\right)\right|=1$.

Then, we deduce: $i \mu\left(G^{\prime}\right)=\alpha(G)+|M|$.

The proof is completed by noticing that the transformation is polynomial.

Then, using exactly the same methodology as in the proof of Theorem 2.4, we show:

Theorem 3.4 MIM is NP-hard in subgrids of degree 2 and 4 and of arbitrarily large girth.

\section{Concluding remarks}

In this paper, we have shown the NP-hardness of MMM and MIM in induced sugrids. Similar thought processes have been applied to other combinatorial problems known to be NP-hard in planar graphs of maximum degree 4, like Independent Dominating Set [6] or List Coloring [8]. It would be interesting to unify all such results or even to derive general conditions allowing us to conclude the NP-hardness in subgrids from NP-hardness in planar graphs of maximum degree 4. A first step is the following proposition. Given a graph $G$ with weights $W$ on its edges, and two integers $r$ and $p$ where $r<p$, we denote by $\widetilde{G}_{W, p, r}$ the graph obtained from $G$ by replacing each edge $u v$ by a path of length $p w(u v)+r$.

Proposition 4.1 Given a problem $\pi$ satisfying:

(1) there are two integers $r$ and $p$ where $r<p$ such that for any graph $G$, any $k$ and $W$, there is $k^{\prime}$ such that

$$
\operatorname{opt}_{\pi}(G) \leq k \Leftrightarrow \operatorname{opt}_{\pi}\left(\widetilde{G}_{W, p, r}\right) \leq k^{\prime}
$$

if $\pi$ is a minimization problem, and

$$
\operatorname{opt}_{\pi}(G) \geq k \Leftrightarrow \operatorname{opt}_{\pi}\left(\widetilde{G}_{W, p, r}\right) \geq k^{\prime}
$$

if $\pi$ is a maximization problem, 
and either

(2) $r(p+r)$ is even and $\pi$ is NP-hard in planar graphs of maximum degree in $D \subset\{1, \ldots, 4\}$,

or

(2') $r$ is odd, $p$ is even and $\pi$ is NP-hard in bipartite planar graphs of maximum degree in $D \subset$ $\{1, \ldots, 4\}$,

then $\pi$ is NP-hard in subgrids of degree in $\{2\} \cup D$ and of arbitrarily large girth.

Proof sketch: Starting from a planar graphs of degree in $D$, the proof consists in embedding it in a grid, subdividing edges to transform it into a partial subgrid, performing a $p^{i}$-expansion for some appropriate $i$ and locally correcting length of $P_{u v}$ such that $\left|P_{u v}\right| \equiv r \bmod p$. As shown in the proof of Theorem 2.4 it is easy to correct the length of each $P_{u v}$ by extending its length by a fixed even number $x$, which allows us to conclude in case (2) by taking $x=r$ or $x=p+r$. In case (2'), we first modify the subgrid after $p^{i}$-expansion as follows (see [8]): let $(A, B)$ be a bipartition of $G$, for any vertex $u_{0}$ of the subgrid corresponding to a vertex of $A$, replace $u_{0}$ by one of its neighbors in the subgrid and make the appropriate modification on $P_{u v}$ for any neighbor $v$ of $u$ in $G$ (see Figure 5). In this way, the length of each $P_{u v}$ is modified by one which allows us to take $x=r \pm 1$. In both cases, the related NP-hardness result in planar graphs gives the conclusion.

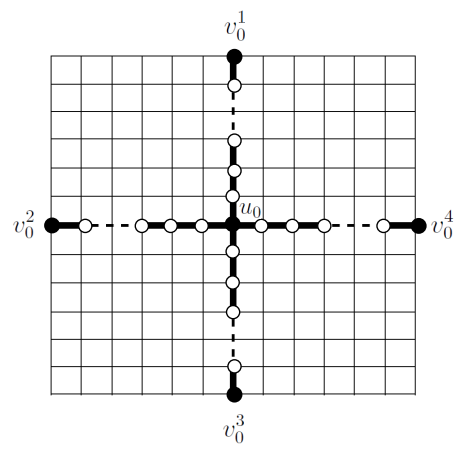

Before modification

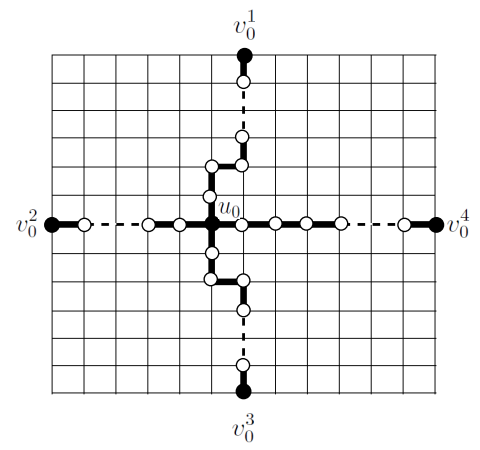

After modification

Fig. 5: Modification of $u_{0}$ corresponding to $u \in A$ in the subgrid in case (2') of Proposition 4.1

Note that for many problems known to be NP-hard in planar graphs of degree 4 and polynomial in subgrids e.g., maximum independent set, such a reduction does not exist. The condition (1) describes an instance transformation where edges are replaced by a gadget that is a path of some specific (arbitrarily large) length. Interesting further work would be to extend Proposition 4.1 using more general gadgets in order to get new NP-hardness results in subgrids. A natural question is then to find which problems known to be NP-hard in bipartite planar graphs are polynomially solvable in subgrids.

Another research direction concerns the complexity of MMM and MIM in grid graphs which have a very regular structure unlike induced subgrids. For MIM, a first step has been done in [19] showing that MIM can be solved in polynomial time in $n \times m$ grids for some cases of $n$ and $m$. For MMM, we conjecture that $\beta\left(G_{n, m}\right)=\left\lceil\frac{n m}{3}\right\rceil$. 


\section{References}

[1] M. Bodur, T. Ekim, and Z. C. Taşkın. Decomposition algorithms for solving the minimum weight maximal matching problem. Networks, 2013. to appear, DOI: 10.1002/net.21516.

[2] K. Cameron. Induced matchings. Discrete Applied Mathematics, 24:97-102, 1989.

[3] K. Cameron. Induced matchings in intersection graphs. Discrete Mathematics, 278:1-9, 2004.

[4] K. Cameron, R. Sritharan, and Y. Tang. Finding a maximum induced matching in weakly chordal graphs. Discrete Mathematics, 266:133-142, 2003.

[5] J.-M. Chang. Induced matchings in asteroidal triple-free graphs. Discrete Applied Mathematics, 132:67-78, 2003.

[6] B.N. Clark and C.J. Colbourn. Unit disk graphs. Discrete Mathematics, 86:165-177, 1980.

[7] K. Dabrowski, M. Demange, and V.V. Lozin. New results on maximum induced matchings in bipartite graphs and beyond. Theoretical Computer Science, 478:33-40, 2013.

[8] M. Demange and D. de Werra. On some coloring problems in grids. Theoretical Computer Science, 472:9-27, 2013.

[9] M. Demange and T. Ekim. Minimum maximal matching is NP-hard in regular bipartite graphs. In Proceedings of 5th International Conference on Theory and Applications of Models of Computation,TAMC 2008, Lecture Notes in Computer Science, volume 4978, pages 364-374, 2008.

[10] W. Espelage, F. Gurski, and E. Wanke. How to solve NP-hard graph problems on clique-width bounded graphs in polynomial time. In Proceedings of the 27th International Workshop on Graph-Theoretic Concepts in Computer Science, WG 2001, Lecture Notes in Computer Science, volume 2204, pages 117-128, 2001.

[11] M.C. Golumbic and R.C. Laskar. Irredundancy in circular-arc graphs. Discrete Applied Mathematics, 44:79-89, 1993.

[12] M.C. Golumbic and M. Lewenstein. New results on induced matchings. Discrete Applied Mathematics, 101:157-165, 2000.

[13] J.M. Harris, J.L. Hirst, and M.J. Mossinghoff. Combinatorics and Graph Theory. Springer, 2nd edition, 2008.

[14] J.D. Horton and K. Kilakos. Minimum edge dominating sets. SIAM Journal of Discrete Mathematics, 6(3):375-387, 1993.

[15] S.F. Hwang and G.J. Chang. The edge domination problem. Discussiones Mathematicae Graph Theory, 15(1):51-57, 1995.

[16] C.W. Ko and F.B. Shepherd. Bipartite domination and simultaneous matroid covers. SIAM Journal on Discrete Mathematics, 16:517-523, 2003.

[17] D. Kobler and U. Rotics. Finding maximum induced matchings in subclasses of claw-free and $p_{5}$-free graphs, and in graphs with matching and induced matching of equal maximum size. Algorithmica, 37:327-346, 2003.

[18] V.V. Lozin. On maximum induced matchings in bipartite graphs. Information Processing Letters, 81:7-11, 2002.

[19] R. Marinescu-Ghemeci. Maximum induced matchings in grids. In Proceedings of 10th Balkan Conference on Operational Research, pages 304-311, 2011.

[20] S.L. Mitchell and S.T. Hedetniemi. Edge domination in trees. In Proceedings of the 8th Southeastern Conference on Combinatorics, Graph Theory and Computing (Louisiana State Univ., Baton Rouge, La., 1977), pages 489-509, 1977.

[21] M.B. Richey and R.G. Parker. Minimum-maximal matching in series-parellel graphs. European Journal of Operations Research, 33(1):98-105, 1988.

[22] A. Srinivasan, K. Madhukar, P. Nagavamsi, C. Pandu Rangan, and M.-S. Chang. Edge domination on bipartite permutation graphs and cotriangulated graphs. Information Processing Letters, 56(3):165-171, 1995.

[23] L.J. Stockmeyer and V.V. Vazirani. NP-hardness of some generalizations of the maximum matching problem. Information Processing Letters, 15(1):14-19, 1982.

[24] Z. C. Taşkın and T. Ekim. Integer programming formulations for the minimum weighted maximal matching problem. Optimization Letters, 6(6):1161-1171, 2012.

[25] R. Uehara. NP-complete problems on a 3-connected cubic planar graph and their applications. Technical Report TWCU-M$0004,1996$.

[26] M. Yannakakis and F. Gavril. Edge dominating sets in graphs. SIAM Journal on Applied Mathematics, 38:364-372, 1980.

[27] L. Yanpei, A. Morgana, and B. Simeone. General theoretical results on recti- linear embedability of graphs. Acta Mathematicae Applicatae Sinica, 7:187-192, 1991.

[28] M. Zito. Randomised Techniques in Combinatorial Algorithmics. PhD thesis, Department of Computer Science,University of Warwick, 1999. 